\title{
Translational Perioperative and Pain Medicine
}

ISSN: 2330-4871

\section{Reevaluating Withholding Intraoperative Opioids: A Lesson Learned from Visceral Pain Control in Laparoscopic Colectomy with TAP Block}

\author{
Jinlei Li ${ }^{1}$, Valeria Possick ${ }^{1}$, Gregory Lessans ${ }^{1}$, John M Aversa ${ }^{2}$ and Feng Dai ${ }^{3}$ \\ ${ }^{1}$ Department of Anesthesiology, School of Medicine, Yale University, USA \\ ${ }^{2}$ Department of Colorectal Surgery, School of Medicine, Yale University, USA \\ ${ }^{3}$ Department of Biostatistics, School of Public Health, Yale University, USA
}

\begin{abstract}
Introduction: An early recovery after surgery (ERAS) program on laparoscopic colectomy with preoperative transversus abdominis plane (TAP) block was initiated to improve post-anesthesia care unit (PACU) turnover and optimize pain control. This study aims to investigate the role of opioids in pain control in such patients.
\end{abstract}

Methods: A retrospective chart review was performed on patients with versus without TAP blocks.

Results: Patients who had preoperative TAP blocks received significantly less long-acting opioids intraoperatively as well as perioperatively with intraoperative and PACU opioids combined ( $p<0.001$ ), but the PACU long-acting opioids requirements were similar between the two groups ( $p$ $=0.96$ ). In addition, this program neither reduced the PACU recovery time nor cut down the length of hospital stays.

Conclusions: Intraoperative opioids are still indicated/indispensable though the requirement may be much lower in certain abdominal procedures in the presence of specific peripheral nerve blockade.

\section{Introduction}

Early recovery after surgery (ERAS) as a form of health care standardization is value-based and evidence-driven. Multiple studies have demonstrated the efficacy of ERAS protocols in colorectal surgeries in the format of less perioperative complications and shorter length of hospital stays [1,2]. Pain control in colorectal surgeries is particularly important as inadequate analgesia has negative impacts on patient satisfaction and ambulation, yet opioids and their documented effects of delaying bowl function returning after surgery can be associated with prolonged length of hospital stays. A trial of ERAS in laparoscopic colectomy was initiated in an academic institute with the goals to: (1) Optimize perioperative pain control by emphasizing opioids sparing techniques and integrating preoperative transversus abdominis plane (TAP) blocks [3]; (2) Improve PACU turn over; (3) Decrease length of hospital stays; (4) Improve patient satisfaction. However, a couple of months into this endeavor, the charge nurse in PACU stated that all TAP blocks in ERAS failed and ERAS patients needed as much opioids as non-ERAS patients and occasionally had even longer stays in PACU. An investigation was initiated immediately for better pain control and improving patient care. The outcomes of our investigation are reported below.

\section{Materials and Methods}

With institutional IRB approval, a retrospective chart review was performed on 40 and 28 patients immediately prior to and after ERAS implementation, respectively. The only management difference between the ERAS vs. non-ERAS group was with vs. without preoperative TAP blocks. All TAP blocks were performed or supervised by a regional anesthesia-fellowship trained attending anesthesiologist under ultrasound guidance. Each EARS patient received $10 \mathrm{ml}$ liposomal bupivacaine plus 10 $\mathrm{ml} 0.25 \%$ bupivacaine on each side. Inclusion Criteria: (1) Elective laparoscopic colectomy; (2) Operated by the same surgeon Dr. J. A. Exclusion Criteria: (1) Chronic usage of opioids, gabapentinoids or any other pain-modulating medications prior to surgery; (2) Contraindications to TAP blocks; (3) Patients who received other type of opioids in addition to fentanyl and hydromorphone (the most commonly used in our institute), or ketamine or dexmedetomidine or NSAIDs, perioperatively; (4) Any perioperative complications during this hospital stay.

\section{Statistical Analysis}

Unless otherwise noted, Wilcoxon rank sum test or Fisher's exact test $p$-value is presented. To handle the excessive zeros (1/40 vs. $18 / 28$ in ESRA vs. non-ESRA) in the data, a finite mixture (logistic-lognormal) model was also fit, in which age, BMI, and gender were adjusted for. All statistical analyses were performed using the statistical software SAS v9.4 (Cary, NC). A Bonferroni-corrected p-value (for 10 multiple tests) of less than 0.05 was considered to be statistically significant. 
Table 1: Comparison of demographic variables by block status.

\begin{tabular}{|c|c|c|c|c|}
\hline & \multicolumn{2}{|c|}{ BLOCK } & \multirow[b]{2}{*}{ Total $(N=68)$} & \multirow[b]{2}{*}{ P Value } \\
\hline & No $(N=40)$ & Yes $(N=28)$ & & \\
\hline \multicolumn{5}{|l|}{ Age } \\
\hline Mean (SD) & 64.93 (11.99) & $61.21(15.86)$ & $63.40(13.73)$ & 0.28 \\
\hline \multicolumn{5}{|l|}{ Weight } \\
\hline Mean (SD) & $84.79(19.50)$ & 79.35 (19.08) & 82.55 (19.37) & 0.26 \\
\hline \multicolumn{5}{|l|}{ BMI } \\
\hline Mean (SD) & $29.30(5.60)$ & $28.15(6.39)$ & $28.83(5.92)$ & 0.44 \\
\hline \multicolumn{5}{|l|}{ Gender } \\
\hline Female & $18(45.00 \%)$ & $12(42.86 \%)$ & $30(44.12 \%)$ & 0.86 \\
\hline Male & $22(55.00 \%)$ & $16(57.14 \%)$ & $38(55.88 \%)$ & \\
\hline
\end{tabular}

Table 2: Opioid consumption and recovery in laparoscopic colectomy patients with TAP block (ERAS) vs. without TAP block (non-ERAS).

\begin{tabular}{|c|c|c|c|c|}
\hline & Non-ERAS: No block & $\begin{array}{l}\text { ERAS: Preoperative } \\
\text { TAP block }\end{array}$ & Original $\mathbf{P}$-value & $\begin{array}{l}\text { Bonferroni } \\
\text { corrected } \\
\text { p-value }\end{array}$ \\
\hline $\mathrm{n}$ & 40 & 28 & & \\
\hline Intraoperative i.v. Fentanyl, mcg, median (IQR) & $200.0(100.0-200.0)$ & $300.0(200.0-350.0)$ & $<0.0001$ & $<0.001$ \\
\hline $\begin{array}{l}\text { Intraoperative i.v. Hydromorphone, mg, } \\
\text { median (IQR) }\end{array}$ & $2.0(1.2-2.0)$ & $0.0(0.0-0.6)$ & $<0.0001^{a}$ & $<0.001$ \\
\hline PACU i.v. Fentanyl, mcg & & & $<0.0001$ & $<0.001$ \\
\hline 0 & 40 & 21 & & \\
\hline 50 & 0 & 3 & & \\
\hline 100 & 0 & 4 & & \\
\hline PACU i.v. Hydromorphone, mg, median (IQR) & $0.4(0.0-1.2)$ & $0.0(0.0-1.1)$ & $0.096^{b}$ & 0.96 \\
\hline $\begin{array}{l}\text { Total perioperative i.v. Hydromorphone, mg, } \\
\text { median (IQR) }\end{array}$ & $2.4(1.9-3.0)$ & $0.63(0-1.8)$ & $<0.0001$ & $<0.001$ \\
\hline $\begin{array}{l}\text { Total opioid consumption during hospital stay, } \\
\text { MME, median (IQR) }\end{array}$ & $74.5(20.0-160.8)$ & $54.8(7.5-143.3)$ & 0.167 & 1 \\
\hline Length of PACU stay, minutes, median (IQR) & $131.0(120.0-180.0)$ & $145.0(120.0-180.0)$ & 0.747 & 1 \\
\hline \multicolumn{5}{|l|}{ First ambulation, days } \\
\hline 0 & 0 & 12 & 0.0003 & 0.003 \\
\hline $1-3$ & 40 & 26 & & \\
\hline Length of hospital stay, days, median (IQR) & $3.0(3.0-5.0)$ & $3.0(2.0-3.5)$ & 0.040 & 0.40 \\
\hline
\end{tabular}

aTo handle the excessive zeros (1/40 vs. 18/28 in ESRA vs. non-ESRA) in the data, a finite mixture (logistic-lognormal) model was also fit, in which age, BMI, and gender were adjusted for. patients with ESRA vs. non-ESRA are found less likely to have any Hydromorphone $(\mathrm{OR}(95 \% \mathrm{Cl})=0.011(0.001-0.102)$, corrected $\mathrm{p}<0.001)$. Of those patients who consumed Hydromorphone, patients with ESRA vs. non-ESRA had significantly less amount (coefficient $(\mathrm{se})=-0.76(0.15)$, corrected $p<0.001$ ).

${ }^{\mathrm{b}}$ To handle the excessive zeros (15/40 vs. 18/28 in ESRA vs. non-ESRA) in the data, a finite mixture (logistic-lognormal) model was also fit, in which age, BMI, and gender were adjusted for patients with ESRA vs. non-ESRA are found less likely to have any Hydromorphone $(\mathrm{OR}(95 \% \mathrm{Cl})=0.247(0.082-0.739), p=0.013)$. Of those patients who consumed Hydromorphone, no statistically significant differences were found between patients with ESRA vs. non-ESRA (coefficient $(\mathrm{se})=-0.195(0.258), p=0.452$ ).

MME: Milligram morphine equivalent.

\section{Results}

There was no statistically significant difference in demographics between the two groups of patients who had the same procedure operated by the same surgeon with an average surgical duration of approximately three hours (Table 1). For short-acting opioids, ERAS patients received statistically more fentanyl than nonERAS intraoperatively [median (IQR): 300 (200-350) mcg vs. 200 (100-200) mcg, Bonferroni corrected $p<0.001$, Table 2. The same trend persisted in PACU. For long-acting opioids, ERAS patients received statistically less hydromorphone than non-ERAS intraoperatively [0.0 (0.0-0.6) mg vs. $2.0(1.2-2.0) \mathrm{mg}$, Bonferroni correct- ed $p<0.001]$. However, this significant difference did not continue in PACU $[0.0$ (0.0-1.1) mg vs. $0.4(0.0-1.2)$ $\mathrm{mg}$, Bonferroni corrected $\mathrm{p}=0.96]$. In addition, total perioperative (intraoperative + PACU) hydromorphone was statistically significant different between ERAS vs. non-ERAS [0.63 (0-1.8) mg vs. 2.4 (1.9-3.0) mg, Bonferroni corrected $p<0.001$ ]. No significant differences between ERAS and non-ERAS were found for PACU stay [145.0 (120.0-180.0) mins vs. 131.0 (120.0-180.0) mins, Bonferroni corrected $p=1.0]$, and for total hospital stay [3.0 (2.0-3.5) days vs. 3.0 (3.0-5.0) days, Bonferroni corrected $p=0.4]$. The total opioids consumption during the entire hospital stay for ERAS vs. non-ERAS were 54.8 
(7.5-143.3) vs. 74.5 (20.0-160.8) milligram morphine equivalent (MME) respectively (Bonferroni corrected $p$ $=1.0$ ). After PACU, while 12 out 28 patients from ERAS group ambulated on the day of surgery, none from the 40 non-ERAS group patients did (Bonferroni corrected $p$ $=0.003$ ). In summary, although ERAS patients received significantly less amounts of long-acting opioids intraoperatively, they required similar amounts of long-acting opioids as non-ERAS in PACU. Consequently, the implementation of EARS protocol by using TAP blocks did not result in quicker PACU turnover. In the author's experience, if these TAP block patients receive a low dose, or as the authors call it, "a background dose", of long-acting opioids intraoperatively, they wake up with minimum discomfort and leave PACU quickly. An alternative option is to re-dose short-acting opioids such as fentanyl throughout the surgery for visceral pain control. It should be reminded that certain peripheral nerve block, such as TAP block, does not provide complete analgesia and patients do still need some, yet significantly less, long-acting or frequent re-dosing of short-acting opioids intraoperatively if visceral pain is anticipated. Otherwise, it will be unfairly left to PACU nurses to catch up on visceral pain control and/or leaving patients in pain.

\section{Discussion}

Most truncal blocks provide effective somatic/incisional pain control, whereas the control for poorly understood visceral pain is provided in descending order as the blockade location moves from the central nervous system to the peripheral nervous system $[4,5]$. Central neuraxial blockade such as spinal anesthesia and epidural anesthesia has the capacity to provide near complete analgesia encompassing both somatic and visceral pain control achieved with local anesthetics with or without a low dose of opioids $[5,6]$. Paravertebral blockade and quadratus lumborum block offer various degrees of visceral pain control for being blocked in the vicinity of the sympathetic trunk. For blocks placed as peripheral as TAP, these techniques certainly provide no visceral analgesia at all. Anesthesiologists therefore should plan accordingly by choosing the most appropriate blockade technique and be prepared to provide necessary supplemental analgesics for the specific surgical procedure involved, be it open or laparoscopic. Surgeons and anesthesiologists need to be reminded that the wide adoption of laparoscopic technique offers a plethora of benefits, but it has failed to decrease persistent postsurgical pain (PPSP). A major independent risk factor for PPSP is poorly controlled acute pain $[7,8]$, hence there are important roles for anesthesiologists to play perioperatively by providing aggressive acute pain control with appropriate regional anesthesia techniques in conjunction with the least necessary amount of opioids [7].
It is known that inadequate acute pain control is not without long-term consequences, rather optimization of acute analgesia has been recognized as essential in chronic pain prevention after surgery or trauma [9]. Opioids, when utilized at proper dosage and for short duration, are indicated rather than contraindicated as one of the potential preventative strategies for chronic pain [10]. It is clear that opioids should not be routinely utilized in chronic non-cancer pain management [11], but this may not be interpreted as denying patients of indicated opioids in acute pain management when there are no other more effective non-opioids options available. Due to the severe consequences of inadequate acute pain control such as chronic pain and significant socioeconomic burden [9], health care providers should avoid simply limiting opioids without providing effective alternatives, which may become yet another "new" underlying cause of poor acute pain management and subsequent chronic pain development. It is about time to critically re-evaluate and differentiate the proper usage of opioids for acute versus chronic pain control during this opioid epidemic and global narcotic shortage [12-14].

\section{References}

1. Nicholson A, Lowe MC, Parker J, Lewis SR, Alderson P, Smith AF. Systematic review and meta-analysis of enhanced recovery programmes in surgical patients. $\mathrm{Br} \mathrm{J}$ Surg. 2014;101(3):172-188.

2. Chemali ME, Eslick GD. A Meta-Analysis: Postoperative Pain Management in Colorectal Surgical Patients and the Effects on Length of Stay in an Enhanced Recovery After Surgery (ERAS) Setting. Clin J Pain. 2017;33(1):87-92.

3. Pirrera $B$, Alagna $V$, Lucchi $A$, et al. Transversus abdominis plane (TAP) block versus thoracic epidural analgesia (TEA) in laparoscopic colon surgery in the ERAS program. Surg Endosc. 2018;32(1):376-382.

4. Cervero F, Laird JM. Visceral pain. Lancet. 1999;353(9170):21452148.

5. Afolayan JM, Olajumoke TO, Amadasun FE, Edomwonyi NP. Intrathecal tramadol versus intrathecal fentanyl for visceral pain control during bupivacaine subarachnoid block for open appendicectomy. Niger J Clin Pract. 2014;17(3):324-330.

6. Alahuhta S, Kangas-Saarela T, Hollmen Al, Edstrom $\mathrm{HH}$. Visceral pain during caesarean section under spinal and epidural anaesthesia with bupivacaine. Acta Anaesthesiol Scand. 1990;34(2):95-98.

7. Gottschalk A, Raja SN. Severing the link between acute and chronic pain: the anesthesiologist's role in preventive medicine. Anesthesiology. 2004;101(5):1063-1065.

8. Joshi GP, Ogunnaike BO. Consequences of inadequate postoperative pain relief and chronic persistent postoperative pain. Anesthesiol Clin North America. 2005;23(1):21-36.

9. Sinatra R. Causes and consequences of inadequate management of acute pain. Pain Med. 2010;11(12):1859-1871.

10. McGreevy K, Bottros MM, Raja SN. Preventing Chronic Pain following Acute Pain: Risk Factors, Preventive Strat- 
egies, and their Efficacy. Eur J Pain Suppl. 2011;5(2):365372 .

11. Olsen Y. The CDC Guideline on Opioid Prescribing: Rising to the Challenge. JAMA. 2016;315(15):1577-1579.

12. Kendall MC, Castro-Alves LJ. Postoperative Pain and Opioid Consumption in the Acute Postoperative Period after Laparoscopic Gastrectomy. J Gastrointest Surg. 2018;22(6):1134.

13. Herzig SJ, Mosher HJ, Calcaterra SL, Jena AB, Nuckols TK. Improving the Safety of Opioid Use for Acute Noncancer Pain in Hospitalized Adults: A Consensus Statement From the Society of Hospital Medicine. J Hosp Med. 2018;13(4):263-271.

14. Urman RD, Boing EA, Khangulov V, et al. Analysis of Predictors of Opioid-Free Analgesia for Management of Acute Post-Surgical Pain in the United States. Curr Med Res Opin. 2018:1-25.
Corresponding Author: Jinlei Li, M.D., Ph.D., Department of Anesthesiology, Yale University, 333 Cedar Street, TMP 3, P.O. Box 208051, New Haven, CT 06520 8051, USA, Tel: 917-601-6828, E-mail: Jinlei.li@yale.edu Editor: Renyu Liu, MD, PhD, Associate Professor, Department of Anesthesiology and Critical Care, Perelman School of Medicine at the University of Pennsylvania, 336 John Morgan building, 3620 Hamilton Walk, Philadelphia, PA 19104, Phone: 2157461485, Email: liur@uphs.upenn.edu

\section{Additional publication details}

Journal short name: Transl Perioper \& Pain Med

Received Date: June 09, 2018

Accepted Date: July 11, 2018

Published Date: July 28, 2018

Citation: Li J, Possick V, Lessan G, et al. Reevaluating Withholding Intraoperative Opioids: A Lesson Learned from Visceral Pain Control in Laparoscopic Colectomy with TAP Block. Transl Perioper \& Pain Med 2018; 5 (3):83-86.

Copyright: (C) $2018 \mathrm{Li}$ J, et al. This is an open-access article distributed under the terms of the Creative Commons Attribution License, which permits unrestricted use, distribution, and reproduction in any medium, provided the original author and source are credited. 